\title{
Pathological characteristics of spine metastases treated with high-dose single-fraction stereotactic radiosurgery
}

\author{
Evangelia Katsoulakis, MD, ${ }^{1}$ llya Laufer, MD, ${ }^{4}$ Mark Bilsky, MD, ${ }^{4}$ Narasimhan P. Agaram, MBBS, ${ }^{2}$ \\ Michael Lovelock, PhD, ${ }^{3}$ and Yoshiya Yamada, MD ${ }^{1}$
}

Departments of ${ }^{1}$ Radiation Oncology, ${ }^{2}$ Pathology, ${ }^{3}$ Medical Physics, and ${ }^{4}$ Neurosurgery, Memorial Sloan Kettering Cancer Center, New York, New York

\begin{abstract}
OBJECTIVE Spine radiosurgery is increasingly being used to treat spinal metastases. As patients are living longer because of the increasing efficacy of systemic agents, appropriate follow-up and posttreatment management for these patients is critical. Tumor progression after spine radiosurgery is rare; however, vertebral compression fractures are recognized as a more common posttreatment effect. The use of radiographic imaging alone posttreatment may make it difficult to distinguish tumor progression from postradiation changes such as fibrosis. This is the largest series from a prospective database in which the authors examine histopathology of samples obtained from patients who underwent surgical intervention for presumed tumor progression or mechanical pain secondary to compression fracture. The majority of patients had tumor ablation and resulting fibrosis rather than tumor progression. The aim of this study was to evaluate tumor histopathology and characteristics of patients who underwent pathological sampling because of radiographic tumor progression, fibrosis, or collapsed vertebrae after receiving high-dose single-fraction stereotactic radiosurgery.

METHODS Between January 2005 and January 2014, a total of 582 patients were treated with linear accelerator-based single-fraction (18-24 Gy) stereotactic radiosurgery. The authors retrospectively identified 30 patients (5.1\%) who underwent surgical intervention for 32 lesions with vertebral cement augmentation for either mechanical pain or instability secondary to vertebral compression fracture $(n=17)$ or instrumentation $(n=15)$ for radiographic tumor progression. Radiation and surgical treatment, histopathology, and long-term outcomes were reviewed. Survival and time to recurrence were calculated using the Kaplan-Meier method.
\end{abstract}

RESULTS The mean age at the time of radiosurgery was 59 years (range $36-80$ years). The initial pathological diagnoses were obtained for all patients and primarily included radioresistant tumor types, including renal cell carcinoma in 7 $(22 \%)$, melanoma in $6(19 \%)$, lung carcinoma in $4(12 \%)$, and sarcoma in $3(9 \%)$. The median time to surgical intervention was 24.7 months (range 1.6-50.8 months). The median follow-up and overall survival for all patients were 42.5 months and 41 months (overall survival range 7-86 months), respectively. The majority of assessed lesions showed no evidence of tumor on pathological review ( 25 of $32,78 \%$ ), while a minority of lesions revealed residual tumor $(7$ of $32,22 \%)$. The median survival for patients after tumor recurrence was 5 months (range 2-70 months).

CONCLUSIONS High-dose single-fraction radiosurgery is tumor ablative in the majority of instances. In a minority of cases, tumor persists and salvage treatments should be considered.

https://thejns.org/doi/abs/10.3171/2016.10.FOCUS16368

KEY WORDS spine stereotactic radiosurgery; image-guided radiation therapy; pathological correlation; radiation fibrosis; tumor ablation

$\mathrm{M}$ ORE than $20 \%$ of cancer patients will develop metastatic disease in the spine. Local control of metastatic spine lesions is accomplished by radiation therapy, surgery, or a combination of both modalities. The actuarial control rates with high-dose single-fraction stereotactic radiosurgery (SRS) are $\geq 90 \% .{ }^{17}$ Advances in systemic therapy have led to improvement in survival for metastatic cancer patients and have made durable control with minimal toxicity imperative for patients. In the longest series to date (median follow-up 6.1 years), long-term survivors post-spinal radiosurgery were identified and analyzed. In that series, spine radiosurgery resulted in a

ABBREVIATIONS IMRT = intensity-modulated radiation therapy; PTV = planning target volume; SRS = stereotactic radiosurgery; SUV = standardized uptake value; VCF = vertebral compression fracture. 
durable 5-year local control rate of more than $90 \%$. Moreover, the safety profile of high-dose spinal radiosurgery was encouraging: there was no Grade 3 or greater toxicity in the long-term survivors. The radiographic vertebral compression fracture (VCF) rate, however, was reported at $36 \% .^{12}$

The most common complication of radiosurgery is VCF. The incidence of VCF varies widely in the literature. Our institution was one of the first to report on VCF incidence, with a radiographic VCF rate of 39\% and median time to VCF of 25 months for 71 lesions treated with single-fraction radiosurgery. ${ }^{14}$ In contrast, in another large series by Boehling et al. of 123 lesions, the VCF rate was $20 \%$ and the median time to VCF was much shorter at 3 months. ${ }^{4}$ In a pooled multiinstitutional analysis examining 410 lesions, the incidence of 1- and 2-year fracture rates was $12.35 \%$ and $13.49 \%$, respectively. The median time to fracture was shorter at 2.46 months. ${ }^{15}$ A major outcome from the study was the impact of dose per fraction on the subsequent development of VCF, namely that the VCF rates at 1 year were $39 \%$ for $\geq 24 \mathrm{~Gy}$ and $10 \%$ for $\leq 19$ Gy. The mechanism underlying VCF may be elucidated by examining the pathological features. The late development of VCF with high-dose single-fraction radiosurgery is thought to develop secondary to radiation as a major contributing factor, specifically fibrosis and necrosis within the tumor and bone that weaken the vertebral body. ${ }^{1}$

The surgical salvage stabilization procedure rate of VCF after radiosurgery varies widely in the literature and is in the range of $11 \%-58 \% .^{14,16}$ The vast majority of patients receive vertebral cement augmentation (kyphoplasty or vertebroplasty), yet up to $25 \%$ may require instrumented surgical intervention for instability.

A small subset of patients, however, requires surgical salvage if there is evidence of radiographic tumor progression. Distinguishing between fibrosis and tumor recurrence in the postradiosurgery setting has significant therapeutic implications. This is the largest reported experience examining the histopathology of post-spinal radiosurgery requiring surgical intervention. In this study, we examine the pathological outcomes, local control, and overall survival in these patients.

\section{Methods \\ Patient Characteristics}

Between January 2005 and January 2014, 582 patients were treated with high-dose single-fraction linear accelerator-based radiosurgery for spinal metastases at Memorial Sloan Kettering Cancer Center. Patients were offered SRS in accordance with the NOMS (neurological, oncological, mechanical, and systemic) multidisciplinary decision framework. ${ }^{2}$ Clinical data including date of radiosurgery and interval between administration of radiosurgery and surgical instrumentation were collected after receiving approval from the institutional review board.

\section{Radiosurgery Technique}

The decision to treat with SRS was made by a multidisciplinary tumor board consisting of spine surgeons, neuroradiologists, and radiation oncologists. The immobiliza- tion and planning technique at Memorial Sloan Kettering Cancer Center has been previously described. ${ }^{10,11}$ Clinical target volumes were delineated using consensus guidelines. An intensity-modulated radiation therapy (IMRT) plan was then generated (planning target volume [PTV]) to treat to a prescription of 18-24 Gy, prescribed to an isodose line that best achieved planning objectives, normalized to $100 \%$. Systemic chemotherapy was discontinued for 1-2 weeks prior to image-guided IMRT. Cone-beam CT was used for image-guided IMRT treatment position verification.

\section{Surgery}

Surgical intervention was considered when VCF resulted in gross spinal instability not amenable to percutaneous cement augmentation or high-grade radiographic epidural spinal cord compression. If surgical instrumentation was not indicated, vertebral cement augmentation was performed in symptomatic patients. Vertebral cement augmentation was used primarily in symptomatic patients with at least $50 \%$ vertebral body collapse, lytic lesions, and without radiculopathy or posterior element disease. In addition, the patient's performance status and extent of metastatic tumor burden were considered when deciding on surgical salvage options.

\section{Follow-Up and Clinical End Points}

After treatment, follow-up imaging was performed using serial MRI. In some instances, PET CT was performed to complement serial MRI. The patients were seen at regular intervals by multidisciplinary teams involving radiation oncologists, neuroradiologists, and spine surgeons. End points examined included radiographic images suggestive of local treatment failure, salvage surgical intervention, pathological characteristics, as well as overall survival.

Patient survival and salvage intervention were calculated from the time of radiation therapy until the last clinic visit or death. Patients typically underwent followup every 12-16 weeks after treatment with image-guided IMRT using routine total spine MR images. Local treatment failure was defined as disease progression within the treated segment of the spine.

\section{Histopathological Analysis}

Specimens obtained at the time of instrumentation surgery as well as vertebral cement augmentation underwent histological examination. All tissue was formalin-fixed, decalcified when necessary, and processed routinely, and $\mathrm{H} \& \mathrm{E}$ stains were prepared. In every case, the study pathologist (N.P.A.) reexamined all slides, and the presence of viable tumor, as well as necrotic tumor, and fibrosis was recorded. In 7 cases the tumor proliferation rate (MIB-1 labeling index) was examined by immunohistochemistry using antibody specific to KI-67 (M7240, 1:200; Dako).

\section{Results}

Between 2005 and 2014, 30 consecutive patients with 32 lesions underwent interventions after SRS as shown 
TABLE 1. Baseline clinical characteristics (overall $n=30$ patients, 32 lesions)

\begin{tabular}{|c|c|}
\hline Parameter & Value \\
\hline \multicolumn{2}{|l|}{ Age at SRS (yrs) } \\
\hline Mean & 59 \\
\hline Range & $36-80$ \\
\hline Median follow-up period (yrs) & 3.5 \\
\hline \multicolumn{2}{|l|}{ Sex } \\
\hline Male & $21(67)$ \\
\hline Female & $11(33)$ \\
\hline \multicolumn{2}{|l|}{ Radiation-resistant metastases } \\
\hline Renal & $7(22)$ \\
\hline Melanoma & $6(19)$ \\
\hline Non-small cell lung & $4(12)$ \\
\hline Sarcoma & $3(9)$ \\
\hline \multicolumn{2}{|l|}{ Radiation-sensitive metastases } \\
\hline Prostate & $3(9)$ \\
\hline Breast & $3(9)$ \\
\hline Other & $6(19)$ \\
\hline \multicolumn{2}{|l|}{ Levels treated } \\
\hline Thoracic & $8(25)$ \\
\hline Thoracolumbar & $4(12)$ \\
\hline Lumbar & $20(62)$ \\
\hline \multicolumn{2}{|l|}{ Radiosurgery dose (Gy) } \\
\hline $24^{*}$ & $29(91)$ \\
\hline $18-22$ & $3(9)$ \\
\hline Median time to intervention (yrs) & 2.1 \\
\hline \multicolumn{2}{|l|}{ Intervention } \\
\hline VCA & $17(53)$ \\
\hline SI & $15(47)$ \\
\hline \multicolumn{2}{|l|}{ Posttreatment pathology } \\
\hline Viable tumor & $7(22)$ \\
\hline Fibrosis & $18(56)$ \\
\hline Fibrosis/necrosis & $7(22)$ \\
\hline
\end{tabular}

$\mathrm{SI}$ = surgical instrumentation; $\mathrm{VCA}=$ vertebral cement augmentation. Values are presented as the number of lesions (\%) unless indicated otherwise. * One patient received radiosurgery at $24 \mathrm{~Gy}$, followed by a second course of repeat radiosurgery at $27 \mathrm{~Gy}$ in 3 fractions, followed by surgical instrumentation with pathology revealing viable tumor.

in Table 1. Interventions included vertebral cement augmentation for VCF or instrumentation for presumed radiographic progression. The mean age at the time of radiosurgery was 59 years (range $36-80$ years). The initial pathological diagnoses were obtained for all patients and primarily included radioresistant histologies, including renal cell carcinoma in 7 (22\%), malignant melanoma in $6(19 \%)$, lung carcinoma in $4(12 \%)$, and sarcoma in $3(9 \%)$. Radiosensitive histologies that received treatment included breast carcinoma in $3(9 \%)$ and prostate carcinoma in $3(9 \%)$. Spinal levels treated included thoracic in 8 patients $(25 \%)$, thoracolumbar in $4(12 \%)$, and lumbar in $20(62 \%)$. The majority of patients $(91 \%)$ received single-fraction high-dose radiosurgery to $24 \mathrm{~Gy}$, with the remaining patients $(9 \%)$ receiving single-fraction 18 - to
TABLE 2. Cases with viable tumor on pathological review postradiosurgery treatment

\begin{tabular}{lccc}
\hline Histology & $\begin{array}{c}\text { Interval to } \\
\text { Intervention } \\
(\mathrm{mos})\end{array}$ & $\begin{array}{c}\text { Radiographic } \\
\text { Progression }\end{array}$ & $\begin{array}{c}\text { Intervention } \\
\text { Type }\end{array}$ \\
\hline Melanoma & 15 & No & VCA \\
\hline Renal, clear cell type & 22 & Yes & SI \\
\hline Leydig cell & 2 & Yes & SI \\
\hline Lung adenocarcinoma & 33 & Yes & SI \\
\hline Melanoma & 2 & Yes & VCA \\
\hline Breast & 39 & Yes & SI \\
\hline Unknown primary & 6 & Yes & SI \\
\hline
\end{tabular}

22-Gy radiosurgery. One of the patients receiving $24 \mathrm{~Gy}$ subsequently received a second course of radiotherapy to $27 \mathrm{~Gy}$ in 3 fractions followed by surgical instrumentation; thus, the cumulative total dose received by 1 patient was $51 \mathrm{~Gy}$. The dosimetry of the treated lesions was satisfactory. The mean PTV was $142 \mathrm{~cm}^{3}$ (range $32.48-368.2 \mathrm{~cm}^{3}$ ). The mean gross tumor volume minimum dose was 16.7 Gy (range 11.3-24.4 Gy), and the mean PTV minimum dose was 14.4 Gy (range 10.6-23.5 Gy).

The median time to intervention was 24.7 months (range 1.6-50.8 months). The majority of lesions necessitated percutaneous cement augmentation techniques (n $=17)$, and the remaining lesions $(\mathrm{n}=15)$ required invasive surgical instrumentation. The majority of salvage procedures $(n=22)$ were performed to treat VCF. Of these, only 1 patient had evidence of viable tumor; otherwise, all other lesions demonstrated fibrosis and/or necrosis. There were 10 lesions with evidence of radiographic tumor progression on MRI that underwent surgical intervention, with only 6 revealing evidence of tumor on pathological review. Only 11 patients (11 lesions) underwent PET CT scanning after radiosurgery and prior to intervention. Of these patients, the majority ( 9 patients) had no evidence of disease pathologically. In these patients, PET CT was equivocal in some instances with a standardized uptake value (SUV) increase observed with compression fractures in the range of 2.2-6.3. In the remaining 2 cases with persistent disease, the available PET CT information was of limited utility. In 1 case, PET was performed 5 months prior to pathological confirmation of disease, and the SUV was only 3.1. In the second case, PET was performed after the second course of radiosurgery and revealed an SUV of only 3.8. In this patient, MRI revealed tumor progression, which was confirmed pathologically 1 month later. A summary of the patient characteristics with residual disease postradiosurgery is shown in Table 2.

The majority of assessed lesions showed complete fibrosis and/or necrosis with no evidence of tumor on pathological review $(\mathrm{n}=25 ; 78 \%)$. A minority of lesions, however, revealed residual tumor $(\mathrm{n}=7 ; 22 \%)$. The median follow-up was 42.5 months, and the median overall survival for the entire cohort was 41 months (range 7-86 months; Fig. 1 upper). The median time to recurrence was 6 months (range 2-39 months). For patients who experienced recurrence, the median overall survival after recur- 

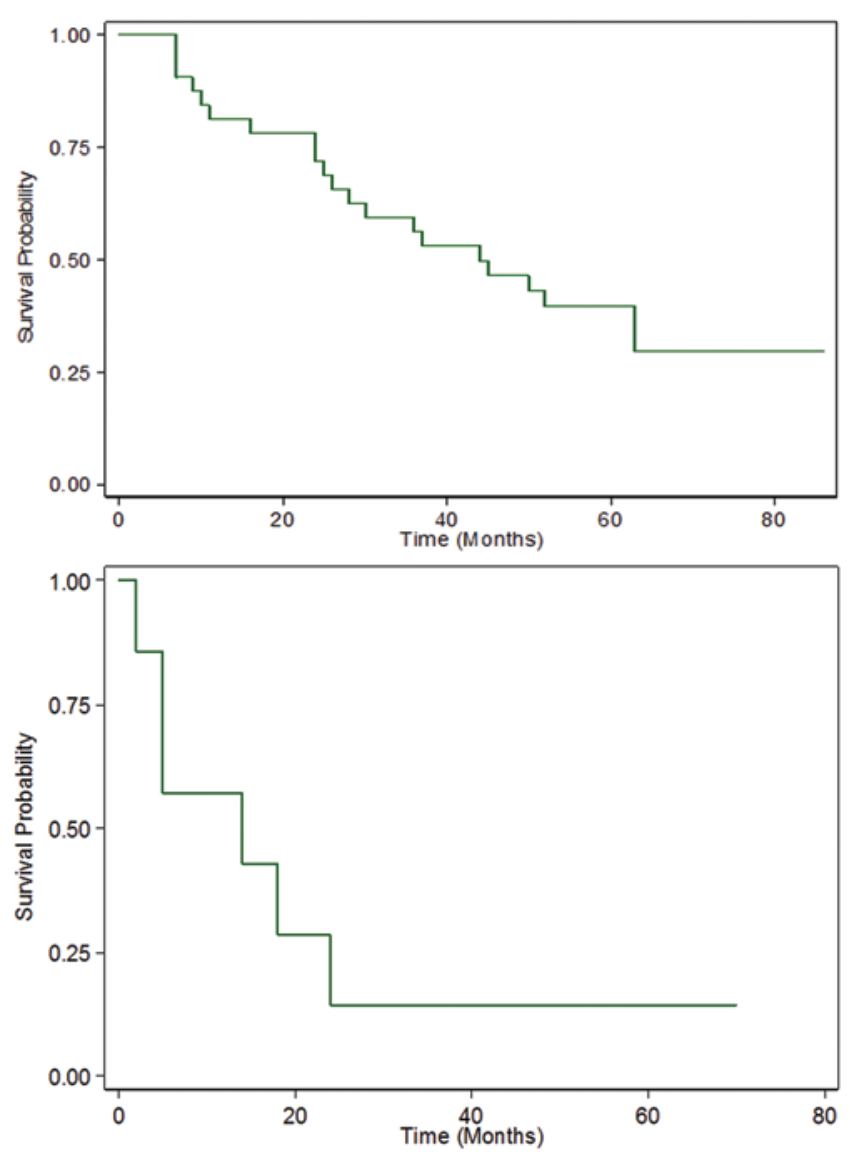

FIG. 1. Upper: Overall survival. Kaplan-Meier analysis for survival in patients treated with single-fraction radiosurgery for spinal metastases who underwent surgical intervention with either vertebral cement augmentation or surgical instrumentation $(n=32)$. The median survival for the entire cohort was 41 months (range 7-86 months). Lower: KaplanMeier analysis for survival in patients treated with single-fraction radiosurgery for spinal metastases that were found to have viable tumor after surgical intervention with either vertebral cement augmentation or surgical instrumentation $(n=6)$. The median overall survival for patients with disease recurrence was 5 months (range 2-70 months).

rence was 5 months (range 2-70 months; Fig. 1 lower). Examples of lesions with viable tumors are shown in Fig. 2B and $\mathrm{D}$. For the pathology specimens revealing no evidence of residual disease, there was evidence of fibrosis $(n=18)$ and necrosis $(n=7)$ as shown in Fig. 2A and C.

\section{Discussion}

It is well established that for radioresistant cancers, the use of single-fraction SRS has resulted in excellent actuarial local control rates of greater than $90 \%{ }^{3,17}$ While the risk of radiation myelopathy is low, the reported rates of VCF are much greater and vary widely from $11 \%$ to $39 \%$ with the median time to VCF ranging from 2 to 25 months. ${ }^{4,5,14}$ The variation in the literature may be partly attributable to the definition of fracture, which includes both fracture progression as well as incidence of new VCFs. In a recent large multiinstitutional pooled analysis of more than 410 patients, the fracture rate was reported to be $14.5 \%$ with a median time to fracture of 2.46 months; however, only $47 \%$ of fractures were new. In addition, while radiographic VCF is concerning and patients often require significantly higher narcotic use, identifying the salvage intervention rate becomes essential. In our previously reported series of fracture risk after SRS, while the overall radiographic VCF rate was $39 \%$, less than $11 \%$ of patients who developed fracture required salvage surgical intervention with either instrumentation or kyphoplasty. This is consistent with the current study in which the surgical intervention rate was only $5 \%$ and acceptable.

While radiographic findings suggestive of VCF progression are straightforward, radiographic findings suggestive of tumor recurrence as the etiology for VCF are not as robust. In this analysis, less than 5\% of VCF cases exhibited active tumor. There are limited guidelines as to the use of biological imaging such as PET that accurately distinguish disease progression from radiation treatment imaging effects. The FDG uptake by tumors on PET CT is variable and largely dependent on tumor histology. The use of MRI alone in determining local recurrence in the spine is less reliable. Just as with radiosurgery of the brain, posttreatment radiation changes may be difficult to discern from tumor recurrence. ${ }^{8}$ In a recent case report by Al-Omair et al., ${ }^{1} 2$ patients underwent tissue sampling after radiographic findings suggested worsening VCF and increased T1 signal intensity on MRI suggested tumor recurrence. In this review, both cases revealed necrotic debris, fibrosis, and avital bone, with 1 case showing fragments of adenocarcinoma. In this series, the significance of tissue sampling was emphasized to differentiate tumor from radiation-induced fibrosis and necrosis, which was attributed as the cause of VCF. In a recent study examining pathological outcomes in patients requiring operative intervention after SRS, viable tumor was found in the periphery of the resected specimens in $60 \%$ of cases. ${ }^{18} \mathrm{In}$ this analysis, 6 of 10 patients who underwent surgery for suspected tumor recurrence were found to have pathological evidence of tumor recurrence; the other cases were found to be fibrosis and/or necrosis only. In our current study, only $22 \%$ of assessed lesions revealed viable tumor. The majority of lesions treated with high-dose single-fraction radiosurgery, however, showed no evidence of tumor on pathological review (78\%), resulting in complete tumor ablation.

It is well established that radiation dose is a significant predictor of local control and potential local cure for spinal metastases. ${ }^{17}$ Prior studies on radiosurgery have demonstrated that a dose greater than $20 \mathrm{~Gy}$ results in high local control rates in the lung, liver, and brain. It is thought that tumor tissue stem cells exposed in vivo to fractions greater than 8-10 Gy respond via a different response mechanism and that high single-dose exposure $(\geq 15 \mathrm{~Gy})$ is linked to the induction of microvascular endothelial apoptosis.? Moreover, in recent studies, radiation dose also appears to have a synergistic role in the immune response. ${ }^{6}$ In preclinical mouse models, while both high-dose radiotherapy in the range of 15-20 Gy $\times 1-3$ fractions and lower dose $3-5$ Gy $\times 4-5$ fractions resulted in higher T-cell infiltration, only high-dose radiotherapy resulted in significantly higher tumor growth delay. ${ }^{13}$ There is increasing interest in harnessing the radioablative doses of radiation to stimulate 


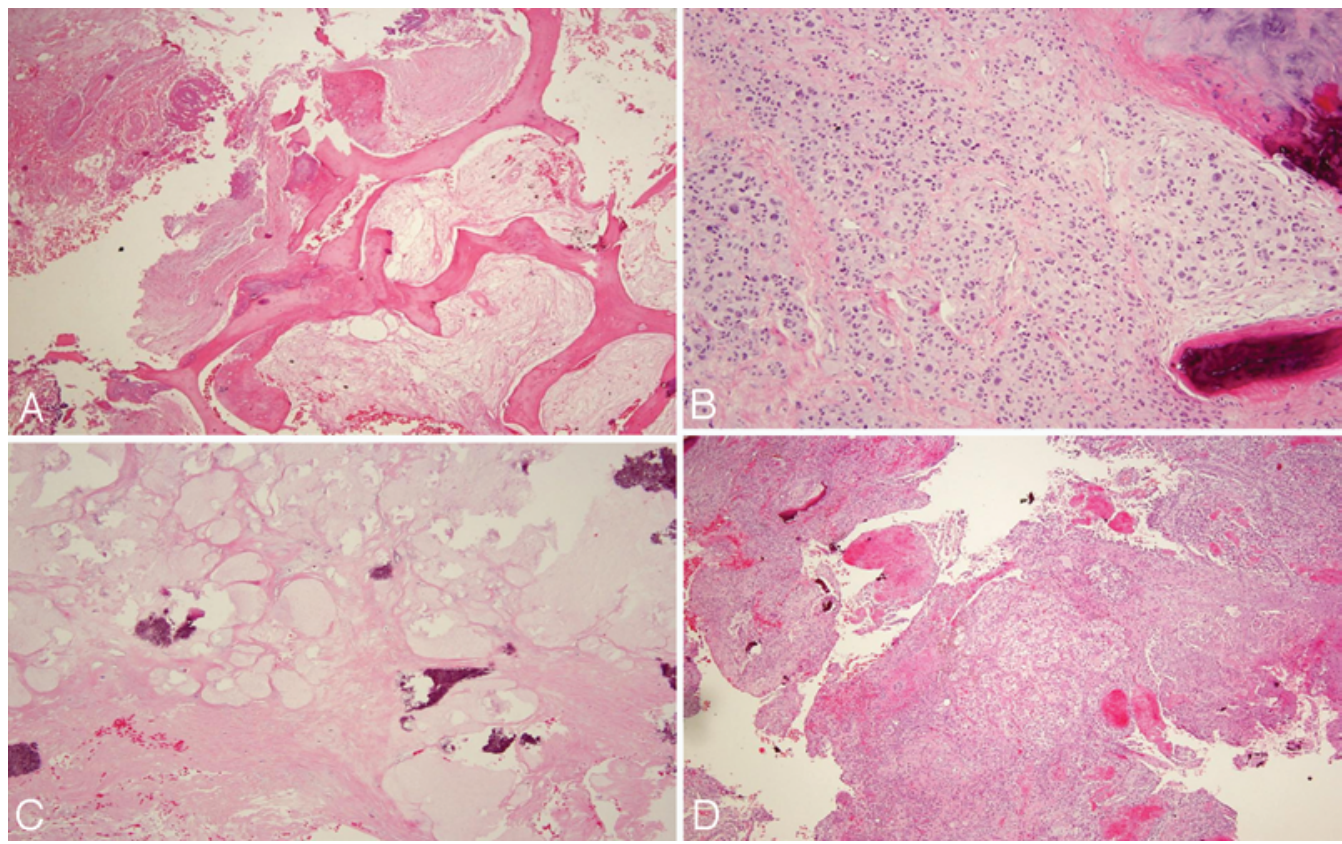

FIG. 2. Photomicrographs of pathology specimens following single-fraction high-dose image-guided radiotherapy. A: Kyphoplasty specimen revealing acellular bone with fibrosis from a patient with melanoma treated with $24 \mathrm{~Gy}$ to L-3. B: Specimen from instrumentation surgery revealing metastatic melanoma for a patient with melanoma treated with 24 Gy to L-5. C: Surgical specimen revealing marrow fibrosis, focal necrosis, and fibroconnective tissue for a patient with renal cell carcinoma treated with 24 Gy to T-9. D: Specimen obtained during instrumentation surgery with viable renal clear cell carcinoma. This patient was treated with 24 Gy to L1-3 followed by additional irradiation 17 months posttreatment with $27 \mathrm{~Gy}$ in 3 fractions. $\mathrm{H}$ \& $\mathrm{E}$, original magnification $\times 100$ $(A, C$, and D); $\times 200(B)$.

the immune system. In a recent prospective randomized study of castrate-resistant metastatic prostate cancer in which patients received single-fraction 8-Gy radiotherapy to bone followed by checkpoint blockade immunotherapy (CTLA4 inhibition), there was suggestion of survival benefit (22.7 months vs 15.8 months [p $=0.0038])$ in a subset of patients with favorable features and lower disease burden including alkaline phosphatase less than 1.5 upper limit of normal and no visceral metastases. ${ }^{9}$ It is highly suggestive that more patients would have responded if radioablative doses of radiation had been administered. This study contributes to the histopathological analyses in the literature confirming that ablation and tumor sterilization comes about postradiosurgery. Future studies are needed to harness the power of radiosurgical tumor ablation in combination with immunotherapy.

Limitations of our study include the small patient number and the possibility of sampling error from cement vertebral augmentation tissue specimens. Nonetheless, this is the largest series reporting on pathological outcomes after SRS to the spine. Our findings suggest that while radiation fibrosis and osteonecrosis may be a contributing factor to VCF, the incidence of salvage surgical interventions in these patients is low and acceptable. Moreover, locally curative radiation therapy and durable tumor ablation are critical via high-dose single-fraction radiosurgery.

\section{Conclusions}

This study demonstrates that SRS is an effective tumor ablative treatment for spinal metastases as evidenced by pathological tissue sampling. The incidence of salvage interventions for symptomatic VCFs is low. The majority of lesions treated had locally ablative, curative, and durable control as evidenced on pathological examination. More accurate methods of radiographic surveillance need to be developed to diagnose local recurrence after spine radiosurgery.

\section{References}

1. Al-Omair A, Smith R, Kiehl TR, Lao L, Yu E, Massicotte EM, et al: Radiation-induced vertebral compression fracture following spine stereotactic radiosurgery: clinicopathological correlation. J Neurosurg Spine 18:430-435, 2013

2. Bilsky M, Smith M: Surgical approach to epidural spinal cord compression. Hematol Oncol Clin North Am 20:1307-1317, 2006

3. Bishop AJ, Tao R, Rebueno NC, Christensen EN, Allen PK, Wang XA, et al: Outcomes for spine stereotactic body radiation therapy and an analysis of predictors of local recurrence. Int J Radiat Oncol Biol Phys 92:1016-1026, 2015

4. Boehling NS, Grosshans DR, Allen PK, McAleer MF, Burton AW, Azeem S, et al: Vertebral compression fracture risk after stereotactic body radiotherapy for spinal metastases. J Neurosurg Spine 16:379-386, 2012

5. Cunha MV, Al-Omair A, Atenafu EG, Masucci GL, Letourneau D, Korol R, et al: Vertebral compression fracture (VCF) after spine stereotactic body radiation therapy (SBRT): analysis of predictive factors. Int J Radiat Oncol Biol Phys 84:e343-e349, 2012

6. Franceschini D, Franzese C, Navarria P, Ascolese AM, De Rose F, Del Vecchio M, et al: Radiotherapy and immunotherapy: Can this combination change the prognosis of patients 
with melanoma brain metastases? Cancer Treat Rev 50:1-8, 2016

7. Garcia-Barros M, Paris F, Cordon-Cardo C, Lyden D, Rafii $\mathrm{S}$, Haimovitz-Friedman A, et al: Tumor response to radiotherapy regulated by endothelial cell apoptosis. Science 300:1155-1159, 2003

8. Jagannathan J, Bourne TD, Schlesinger D, Yen CP, Shaffrey ME, Laws ER Jr, et al: Clinical and pathological characteristics of brain metastasis resected after failed radiosurgery. Neurosurgery 66:208-217, 2010

9. Kwon ED, Drake CG, Scher HI, Fizazi K, Bossi A, van den Eertwegh AJ, et al: Ipilimumab versus placebo after radiotherapy in patients with metastatic castration-resistant prostate cancer that had progressed after docetaxel chemotherapy (CA184-043): a multicentre, randomised, double-blind, phase 3 trial. Lancet Oncol 15:700-712, 2014

10. Laufer I, Iorgulescu JB, Chapman T, Lis E, Shi W, Zhang Z, et al: Local disease control for spinal metastases following "separation surgery" and adjuvant hypofractionated or highdose single-fraction stereotactic radiosurgery: outcome analysis in 186 patients. J Neurosurg Spine 18:207-214, 2013

11. Lovelock DM, Hua C, Wang P, Hunt M, Fournier-Bidoz N, Yenice K, et al: Accurate setup of paraspinal patients using a noninvasive patient immobilization cradle and portal imaging. Med Phys 32:2606-2614, 2005

12. Moussazadeh N, Lis E, Katsoulakis E, Kahn S, Svoboda M, DiStefano NM, et al: Five-year outcomes of high-dose singlefraction spinal stereotactic radiosurgery. Int J Radiat Oncol Biol Phys 93:361-367, 2015

13. Patel KR, Lawson DH, Kudchadkar RR, Carthon BC, Oliver DE, Okwan-Duodu D, et al: Two heads better than one? Ipilimumab immunotherapy and radiation therapy for melanoma brain metastases. Neuro Oncol 17:1312-1321, 2015

14. Rose PS, Laufer I, Boland PJ, Hanover A, Bilsky MH, Yamada J, et al: Risk of fracture after single fraction image-guided intensity-modulated radiation therapy to spinal metastases. J Clin Oncol 27:5075-5079, 2009

15. Sahgal A, Atenafu EG, Chao S, Al-Omair A, Boehling N,
Balagamwala EH, et al: Vertebral compression fracture after spine stereotactic body radiotherapy: a multi-institutional analysis with a focus on radiation dose and the spinal instability neoplastic score. J Clin Oncol 31:3426-3431, 2013

16. Sung SH, Chang UK: Evaluation of risk factors for vertebral compression fracture after stereotactic radiosurgery in spinal tumor patients. Korean J Spine 11:103-108, 2014

17. Yamada Y, Bilsky MH, Lovelock DM, Venkatraman ES, Toner S, Johnson J, et al: High-dose, single-fraction imageguided intensity-modulated radiotherapy for metastatic spinal lesions. Int J Radiat Oncol Biol Phys 71:484-490, 2008

18. Zwagerman NT, McDowell MM, Hamilton RL, Monaco EA III, Flickinger JC, Gerszten PC: Histopathological examination of spine tumors after treatment with radiosurgery. Neurosurg Focus 41(2):E14, 2016

\section{Disclosures}

The authors report the following. Dr. Laufer: consultant for Globus, DePuy/Synthes, and SpineWave. Dr. Yamada: consultant for Varian Medical Systems and medical advisory board member for the Chordoma Foundation.

\section{Author Contributions}

Conception and design: Yamada, Katsoulakis, Laufer, Bilsky. Acquisition of data: Yamada, Katsoulakis, Laufer, Bilsky. Analysis and interpretation of data: all authors. Drafting the article: Yamada, Katsoulakis. Critically revising the article: all authors. Reviewed submitted version of manuscript: all authors. Approved the final version of the manuscript on behalf of all authors: Yamada. Statistical analysis: Katsoulakis, Laufer, Lovelock. Administrative/technical/material support: Katsoulakis, Bilsky. Study supervision: Yamada, Laufer, Bilsky. Pathology review: Agaram.

\section{Correspondence}

Yoshiya Yamada, Department of Radiation Oncology, Memorial Sloan Kettering Cancer Center, 1275 York Ave., New York, NY 10065. email: yamaday@mskcc.org. 